\title{
Encoding of amplitude modulations by auditory neurons of the locust: influence of modulation frequency, rise time, and modulation depth
}

\author{
Sandra Wohlgemuth - Astrid Vogel • \\ Bernhard Ronacher
}

Received: 5 May 2010/Revised: 2 September 2010/Accepted: 6 September 2010/Published online: 24 September 2010

(C) The Author(s) 2010. This article is published with open access at Springerlink.com

\begin{abstract}
Using modulation transfer functions (MTF), we investigated how sound patterns are processed within the auditory pathway of grasshoppers. Spike rates of auditory receptors and primary-like local neurons did not depend on modulation frequencies while other local and ascending neurons had lowpass, bandpass or bandstop properties. Local neurons exhibited broader dynamic ranges of their rate MTF that extended to higher modulation frequencies than those of most ascending neurons. We found no indication that a filter bank for modulation frequencies may exist in grasshoppers as has been proposed for the auditory system of mammals. The filter properties of half of the neurons changed to an allpass type with a 50\% reduction of modulation depths. Contrasting to reports for mammals, the sensitivity to small modulation depths was not enhanced at higher processing stages. In ascending neurons, a focus on the range of low modulation frequencies was visible in the temporal MTFs, which describe the temporal locking of spikes to the signal envelope. To investigate the influence of stimulus rise time, we used
\end{abstract}

Electronic supplementary material The online version of this article (doi:10.1007/s00359-010-0587-4) contains supplementary material, which is available to authorized users.

S. Wohlgemuth · A. Vogel · B. Ronacher $(\bowtie)$

Department of Biology, Humboldt-Universität zu Berlin, Invalidenstrasse 43, 10115 Berlin, Germany

e-mail: bernhard.ronacher@rz.hu-berlin.de

B. Ronacher

Bernstein Center for Computational Neuroscience Berlin,

Unter den Linden 6, 10099 Berlin, Germany

Present Address:

S. Wohlgemuth

Department of Animal Behaviour, Institute of Biology,

Freie Universität, Berlin, Germany rectangularly modulated stimuli instead of sinusoidally modulated ones. Unexpectedly, steep stimulus onsets had only small influence on the shape of MTF curves of $70 \%$ of neurons in our sample.

Keywords Acoustic communication - Modulation transfer functions $\cdot$ Stimulus rise time $\cdot$ Temporal resolution

$\begin{array}{ll}\text { Abbreviations } & \\ \text { AM } & \text { Amplitude modulation } \\ \text { BMF } & \text { Best modulation frequency } \\ \text { MF } & \text { Modulation frequency } \\ \text { rMTF, tMTF } & \text { (rate and temporal) Modulation transfer } \\ & \text { function } \\ \text { SAM } & \text { Sinusoidal amplitude modulation } \\ \text { RAM } & \text { Rectangular amplitude modulation } \\ \text { SPL } & \text { Sound pressure level }\end{array}$

\section{Introduction}

In many taxa, e.g. mammals, birds, frogs, and insects, acoustic communication signals serve to attract conspecific mates and to assess their quality (Kroodsma and Miller 1996; Gerhardt and Huber 2002; Greenfield 2002). The correct interpretation of these sounds contributes directly to the mating success and the production of offspring. Hence, we can expect that strong selection pressures drive the neuronal processing of communication signals. The auditory systems of most insects are characterized by a poor capacity to resolve carrier frequencies (for review, see Hennig et al. 2004). In line with this limitation usually the 
pattern of amplitude modulations (AM) is crucial for signal recognition, while the carrier frequency content plays only a secondary role (Heller 1988; von Helversen and von Helversen 1997; Gerhardt and Huber 2002; Stumpner and von Helversen 2001). The communication signals ('songs') of different grasshopper species, for example, show a great variety in their temporal patterns, while their carrier frequency spectra overlap largely (Elsner 1974; Stumpner and von Helversen 1994; Meyer and Elsner 1996). This implies that the respective receivers are able to process and resolve the rapid amplitude fluctuations of sound patterns.

We investigated how the amplitude modulations of sound signals are processed and represented within the auditory pathway of acridid grasshoppers. As a basic approach, we applied the paradigm of modulation transfer functions (MTF), a well-established method to characterize the processing and filtering capacities of sensory systems. By varying the frequency of sinusoidal amplitude modulations (SAM) one may explore filter properties of neurons, i.e. preferred frequency ranges, as well as the upper limits of temporal resolution (for review, see Joris et al. 2004). The two basic variables of SAM stimuli are modulation frequency and modulation depth. However, a change in modulation frequency or modulation depth also influences the steepness of amplitude rise. Onset steepness of acoustic stimuli is a factor that strongly influences auditory thresholds, spike latencies, and the precision of spiking responses of auditory neurons (Krahe and Ronacher 1993; Heil and Neubauer 2001; Machens et al. 2001; Rokem et al. 2006). Hence, variations in rise time may affect the filter properties of neurons in an unpredictable way (Hall and Feng 1991). We aimed at disentangling rise time from frequency effects by comparing the neuron's responses to sinusoidal (SAM) and to rectangularly modulated (RAM) broadband stimuli.

Electrophysiological recordings were performed on locusts (Locusta migratoria), a species that relies only little if at all on acoustic signalling in the context of mate recognition. This report nevertheless aims at interpreting the results in relation to behavioural data obtained in another species, Chorthippus biguttulus. This attempt is based on a recent study, which provided quantitative evidence that the peripheral auditory system of the two species is highly conserved, and homologous thoracic neurons show highly similar physiological responses in both species (Neuhofer et al. 2008, in preparation; see also Ronacher and Stumpner 1988; Sokoliuk et al. 1989). Based on this physiological similarity, data obtained on locust neurons can be related to the extensive behavioural data set available in $C$. biguttulus (e.g. von Helversen 1972, 1993; von Helversen and von Helversen 1997, 1998; Balakrishnan et al. 2001). These behavioural studies revealed that the temporal pattern of amplitude modulations is crucial for mate recognition in
C. biguttulus. Important cues for recognition are the frequencies of amplitude modulations, with particular emphasis of modulation frequencies between 10 and $150 \mathrm{~Hz}$ (von Helversen and von Helversen 1998; Schmidt et al. 2008; Clemens et al. 2010), modulation depth, duty cycle, and the rise time of sound pulses (von Helversen 1993; Ronacher and Krahe 2000). We tried to cover a large part of this feature space in the design of our stimulation protocols.

For the evaluation of spike responses of auditory neurons to the MTF stimulation paradigm one can focus on two different time scales, yielding rate MTF and temporal MTF (rMTF and tMTF): for the first, the rMTF, the average spike rate is determined over a larger segment of the stimulus, e.g. $500 \mathrm{~ms}$. The tMTF, in contrast, quantifies how precisely the spikes do lock to the modulations of the sound envelope (e.g. Rhode and Greenberg 1994; Krishna and Semple 2000; Joris et al. 2004). These data evaluation procedures correspond to two basic aspects of coding in nervous systems, i.e. spike rate or spike timing codes (Rieke et al. 1996).

Auditory receptor neurons of locusts respond with uniform spike rates to SAM stimuli in a broad frequency range (10-500 Hz); their spiking rate does not depend on the AM frequency, resulting in an allpass type rMTF (Weschke and Ronacher 2008). Accordingly, receptor neurons convey information by the timing of spikes - in their instantaneous spike rate modulation, they preserve to some extent the envelope fine structure of incoming acoustic signals. tMTFs of receptor neurons are mostly bandpass, with a tendency to lowpass behaviour at low sound intensities; the corner frequencies, which are a measure of temporal resolution, reach 150-275 Hz (Prinz and Ronacher 2002; Weschke and Ronacher 2008).

Here, we ask how the temporal information of AM patterns is encoded at the next stages of the auditory pathway. The auditory pathway of acridid grasshoppers is hierarchically organized and consists of three consecutive stages of processing: receptor neurons, local interneurons (LN), and ascending interneurons (AN) (Stumpner and Ronacher 1994; Vogel et al. 2005; Vogel and Ronacher 2007). The latter transmit the pre-processed information to downstream processing centres located in the animal's brain (Römer and Marquart 1984; Ronacher et al. 1986; Stumpner et al. 1991). Grasshoppers have only a small repertoire of auditory neurons to solve similar tasks as vertebrates with their much larger nervous systems. Hence, we can expect that specific filter properties are boiled down to the level of a few or even single neurons (e.g. Ronacher and Stumpner 1988). In this report, we focus on the following questions: How is information about different modulation frequencies processed and transmitted to the brain? What are the limits of temporal resolution at 
different stages of processing? To what degree does onset steepness affect the shape of modulation frequency filters? Can we find neurons that are specialized to certain AM frequency ranges, indicative of a neuronal fractionation of the AM frequency scale into different frequency bands, like the AM filter bank concept proposed for vertebrates (Langner and Schreiner 1988; Joris et al. 2004)? Are amplitude contrasts enhanced at higher levels of processing as proposed for the auditory system of vertebrates (e.g. Malone et al. 2010)?

\section{Materials and methods}

\section{Animals and electrophysiology}

All experiments were performed on adult locusts (Locusta migratoria), which were purchased from a commercial supplier. Intracellular recordings were obtained from auditory receptors, local and ascending interneurons in the frontal auditory neuropil of the metathoracic ganglion. We used standard electrophysiological techniques and equipment (for details see Vogel et al. 2005; Wohlgemuth and Ronacher 2007). During the experiments, the preparation was kept at a constant temperature of $30 \pm 2^{\circ} \mathrm{C}$. At the end of an experiment, the cell was stained with Lucifer Yellow and identified according to their characteristic morphology (terminology after Römer and Marquart 1984; Stumpner and Ronacher 1991). We present data from a total of 55 neurons, which can be divided into four classes: 11 auditory receptors (all of the low frequency type); 11 local neurons with primary-like responses (7 TN1, $3 \mathrm{SN} 1$, and $1 \mathrm{SN} 4$ ); 11 LN with not primary-like responses (1 SN2, 1 SN8, and 9 BSN1); 22 ascending neurons (5 AN1, 1 AN2, 5 AN3, 6 AN4, 3 AN11, 1 AN12, and 1 AN542). According to its morphology, TN1 is a t-fibre sending axon collaterals to the abdominal and all thoracic ganglia. However, due to its primary-like responses and the fact that its axon does not ascend to the brain (Stumpner and Ronacher, unpublished observations), we counted TN1 among the local neurons.

\section{Acoustic stimulation}

The paradigm of modulation transfer functions has the advantage that various stimulus properties can be modified independently in contrast to the 'mix-up' usually found in natural signals. Also many behavioural tests in another grasshopper species, Chorthippus biguttulus, were performed with artificial song models in order to separate signal features that are relevant for signal recognition (von Helversen and von Helversen 1997). Studies using combinations of Fourier components revealed that various combinations of components between 10 and $50 \mathrm{~Hz}$ were sufficient to enable signal recognition in $C$. biguttulus (Schmidt et al. 2008), while higher frequencies are important for sex discrimination and rejection of lowquality songs (von Helversen and von Helversen 1998; see also Kriegbaum 1989).

All experiments were performed in a Faraday cage lined with foam prisms to attenuate echoes. Acoustic stimuli were broadcast via one of two speakers (D-28/2, Dynaudio), situated laterally at a distance of $30 \mathrm{~cm}$ from the preparation. The signals were amplified (Mercury, 2000, Jensen) and attenuated (PA5, Tucker Davis). All acoustic stimuli were stored digitally and delivered by custom-made software (Labview, National Instruments) using a 100-kHz D/A-conversion (PCI-MIO-16E-1, National Instruments). Sound intensities were calibrated with a Brüel and Kjaer microphone (1/2 in.), positioned at the site of the preparation, a Brüel and Kjaer measuring amplifier (type 2209), and are given in $\mathrm{dB}$ re $2 \times 10^{-5} \mathrm{~N} \mathrm{~m}^{-2}$ (dB SPL). The sound intensity of test stimuli was adjusted to $\sim 20 \mathrm{~dB}$ above threshold of the recorded neuron; most neurons were tested with sound intensities in the range of 55-70 dB SPL. The acoustic stimuli used in this study (compare Fig. 1) were sinusoidal amplitude modulations (10, 20, 40, 83, 125, 167, 250, 333, and $500 \mathrm{~Hz}$; modulation depth 100\%) of a broad-band noise carrier $(0.5-30 \mathrm{kHz})$. Stimuli were generated in the Labview programming environment (National Instruments). Each SAM stimulus was preceded by a 200-ms segment of unmodulated noise, followed by a 1-s segment of constant modulation frequency and depth, and another segment of $200 \mathrm{~ms}$ of unmodulated noise. The stimuli were repeated four times with stimulus intervals of $300 \mathrm{~ms}$. For a few cells, another set of stimuli was used, which had a 4-s segment of constant modulation frequency while all other stimulus parameters were identical. These stimuli were only repeated once. For the comparison of sinusoidally and rectangularly modulated stimuli (see below), the stimuli had $500 \mathrm{~ms}$ duration, without adaptation pulse, and were repeated eight times. No systematic differences were observed in the data analysis between the 500-ms, 1-s, and the 4-s stimuli. 15 cells were stimulated with both sinusoidally and rectangularly modulated stimuli (SAM and RAM stimuli). A few of these neurons were also tested with intermediate rise times that were generated according to Eq. 1-see "Results".

$f(x)=\left(\frac{\tanh [-\beta \times \cos (x)]}{\tanh (\beta)}+1\right) \times 1 / 2$,

with $\beta=0.001$ for sinusoidally modulated (SAM) stimuli and $\beta=1,000$ for rectangularly modulated stimuli (RAM). 
Fig. 1 rMTF and tMTF curves of a TN1 neuron. Shown are peri-stimulus-time histograms (PSTHs) for modulation frequencies between 10 and $500 \mathrm{~Hz}$. Spike rates (red solid line and squares, upper scale) and vector strengths (green dashed line, triangles, lower scale) are shown for the respective frequencies. On the left, the morphology of the TN1 neuron is depicted

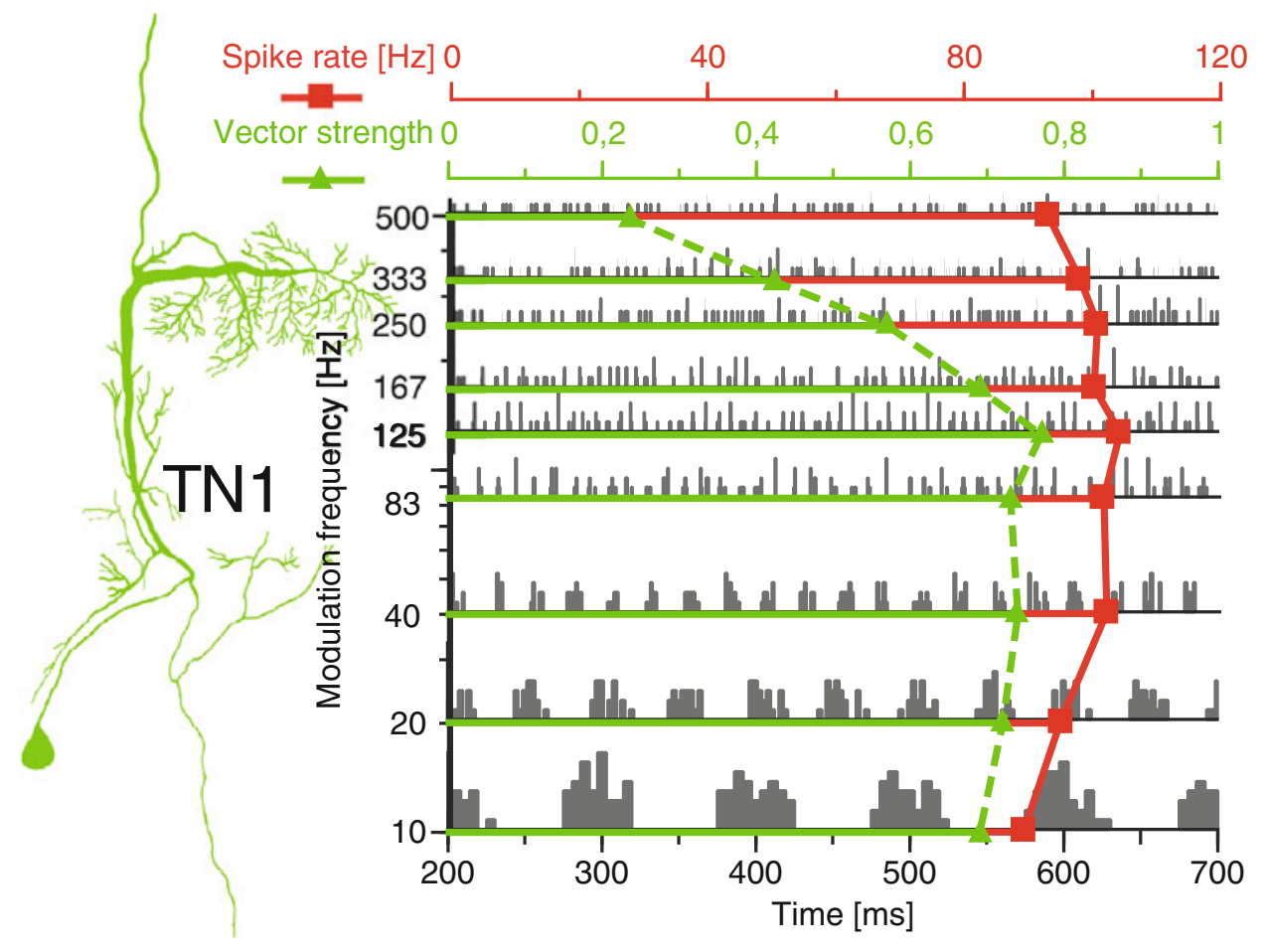

The two procedures are illustrated in Fig. 1 on the example of a TN1 neuron. Shown in this figure are nine peri-stimulus-time histograms (PSTH) for the different frequencies of sinusoidal amplitude modulations for a time segment of $500 \mathrm{~ms}$. AM depth was $100 \%$ in this case. Above the PSTHs are two scales: the red one for spike rate, green for vector strength. The red, solid curve describes the spike rates, averaged over the whole $500 \mathrm{~ms}$ segment of the PSTH. The green, dashed curve refers to the respective vector strength. For this neuron, the rate MTF curve corresponds to an allpass filter, while the vector strength curve belongs to the lowpass filter type.

The MTF based on rate (rMTF) were determined by plotting the spike rate averaged over the four repetitions of the SAM part of the stimulus against the modulation frequency. A rate filter property, i.e. a deviation from an allpass filter, was recognized if spike rates of at least two consecutive modulation frequencies deviated by at least $40 \%$ from the average spike rate of that cell (Franz 2004). We chose this conservative criterion to reduce the influence of obviously random spike rate fluctuations as some rMTFs had a jagged appearance (see also Hall and Feng 1991; Weschke and Ronacher 2008).

To obtain vector strength-based modulation transfer functions (tMTF), period histograms were generated from the SAM part of the stimulus (see Fig. 1). Each period histogram was divided into 30 bins, i.e. bins cover different absolute time scales depending on the modulation 
frequency. From these period histograms, the vector strength was calculated using Eq. 2:

$\mathrm{VS}=\sqrt{\left(\frac{1}{n} \sum_{i=1}^{n} \cos \alpha_{i}\right)^{2}+\left(\frac{1}{n} \sum_{i=1}^{n} \sin \alpha_{i}\right)^{2}}$,

with $\alpha_{i}$ denoting spike times expressed as the phase of stimulus. We calculated Rayleigh's $\mathbf{z}$ to control for the significance of phase locking. A value of $\mathbf{z}=3$, which corresponds to a 5\% significance level, was chosen as threshold (Gleich and Klump 1995; Prinz and Ronacher 2002). Only at high modulation frequencies $(250 \mathrm{~Hz}$ or higher) Rayleigh's $\mathbf{z}$ regularly fell below this threshold criterion. Therefore, phase locking of spikes was significant in most tMTFs up to $167 \mathrm{~Hz}$. tMTFs are shown as vector strengths at $100 \%$ modulation depth. Following Krishna and Semple (2000), corner frequencies of tMTFs were determined as the modulation frequency where the vector strength dropped to $90 \%$ of its maximal value.

\section{Statistics}

Data from the four classes of neurons (receptors, primarylike local neurons, other local neurons, and ascending neurons) were compared using an ANOVA combined with the Tukey-Kramer post hoc test, or in case of not normal distributed data with the Kruskal-Wallis non-parametric ANOVA and Dunn's post hoc test.

\section{Results}

From the neurons recorded, we show rate-MTF only for a few examples that cover the main filter types. To demonstrate the variability within and between neuron types, a large sample of specimens is shown as supplementary Fig. 1. Receptors and the local neurons with primary-like responses (see "Materials and methods" for classification) exhibited allpass rate-MTF, with only two exceptions (see Fig. 2, left; supplementary Fig. 1). The BSN1 is a local neuron that occurs as twin cells with slightly different response properties (Römer and Marquart 1984; Stumpner 1989). The rMTF of six BSN1 specimens belonged to the lowpass type (Fig. 2), while three exhibited a tendency to allpass or bandpass. Among the ascending neurons, we found mostly lowpass and bandstop filters (Fig. 2) and, rarely, highpass types. In order to visualize the basic properties of a large sample of cells, we decided to extract two characteristic features of the rMTF curves-illustrated in the scheme in Fig. 3: the difference between maximal and minimal spike rate indicates whether a neuron belongs to the allpass type. Our criterion for classifying responses as deviating from the allpass type was conservative, i.e. a deviation of at least $40 \%$ from the maximal spike rate (see "Materials and methods"). Applying this criterion, most specimens of BSN1 and ascending neurons exhibited filter properties, i.e. stronger responses within a certain frequency range, while receptors, TN1, and most other primary-like neurons did not. For neurons with clear filter properties, we plotted the dynamic range on the frequency axis, i.e. the range of frequencies where the response dropped from $10 \%$ below the maximum to $10 \%$ above the minimum (Fig. 3b, see inset for definition). This dynamic range is of interest since it marks a neuron's potential for signalling distinct $\mathrm{AM}$ frequency ranges to downstream neurons via changes in firing rates (see "Discussion"). For neurons with band stop filter characteristic (as in the right diagram of Fig. 2), only the dynamic range of the left hand side of the curve is depicted in Fig. 3b; also shown is the worst modulation frequency (WMF), i.e. the position of the minimum response. Different specimens of some neuron types (AN1 and AN3) showed quite some variability in their dynamic ranges, while others showed similar dynamic ranges (AN4 and AN11). Nonetheless, a tendency is visible in Fig. $3 \mathrm{~b}$ that most ascending neurons had narrower dynamic ranges compared to BSN1 and SN2. Thus, a change in spike rate of an ascending neuron could indicate a more restricted AM-frequency segment compared to local neurons.

As the next step, we analysed how the sound envelope information is preserved in the spike responses at higher processing stages by calculating temporal MTFs. The tMTF of all neurons included in this study showed lowpass
Fig. 2 Rate MTF curves of two local and four ascending neurons. Abscissa modulation frequency $(\mathrm{Hz})$; ordinate spike rate $(\mathrm{Hz})$
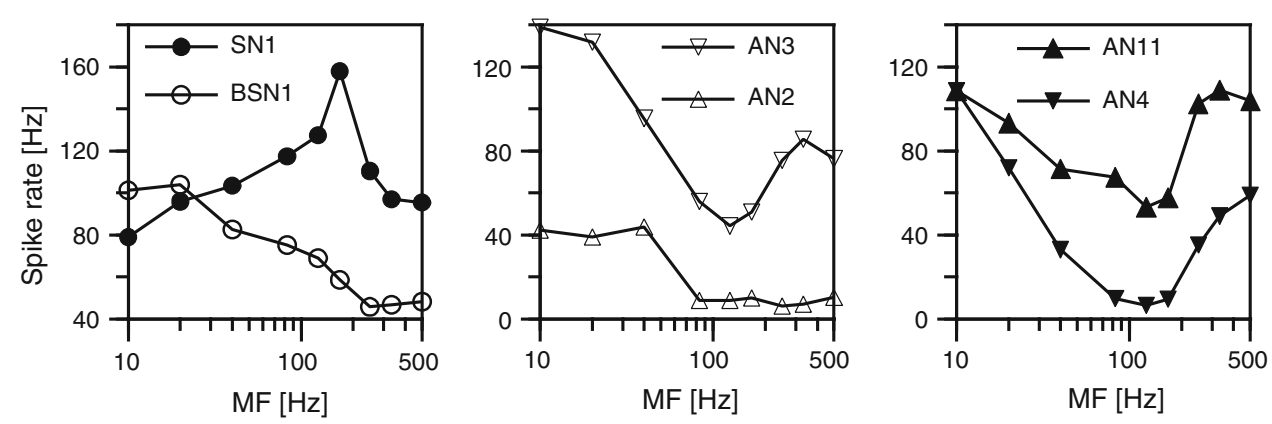
Fig. 3 Summary diagram of rMTF data. a Difference between maximal and minimal spike rate as an indicator of filter properties (for definitions see inset). $R$ receptors, $L N$ local neurons, and $A N$ ascending neurons. b Dynamic ranges of different neurons. For bandstop filters the position of the lowest response $(W M F)$ is also indicated. Receptor neurons and primary-like neurons with allpass properties are not included in b. Neuron types according to Römer and Marquart (1984) and Stumpner and Ronacher (1991)

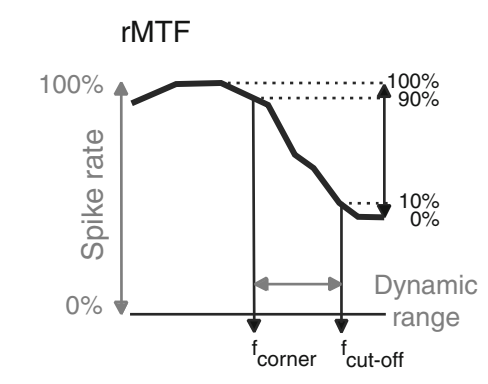

a

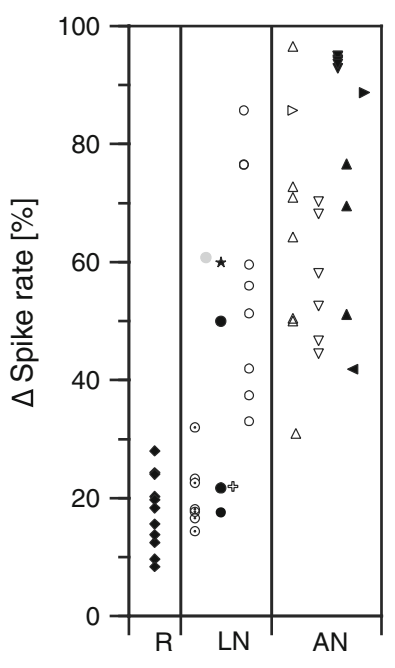

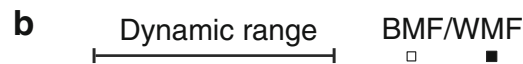
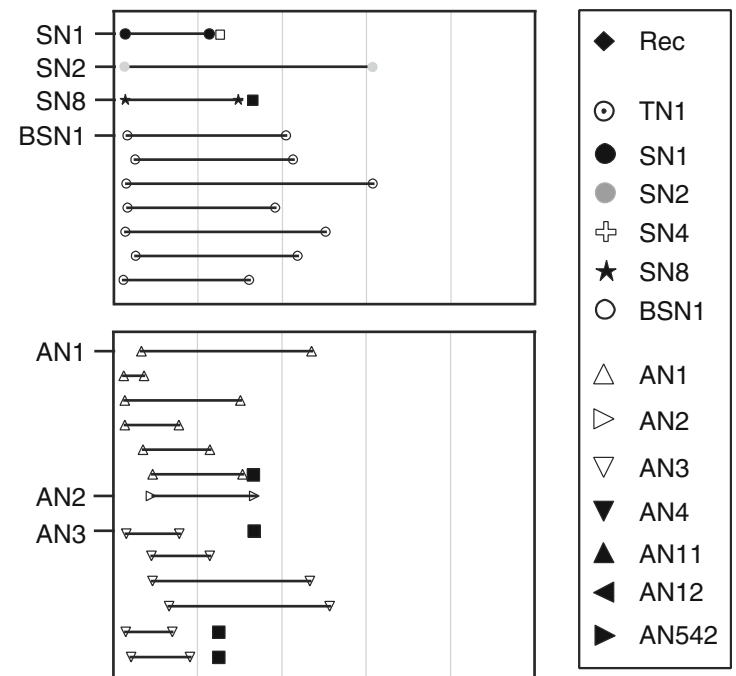

Fig. 4 Sample of tMTF curves (same neurons as in Fig. 2)
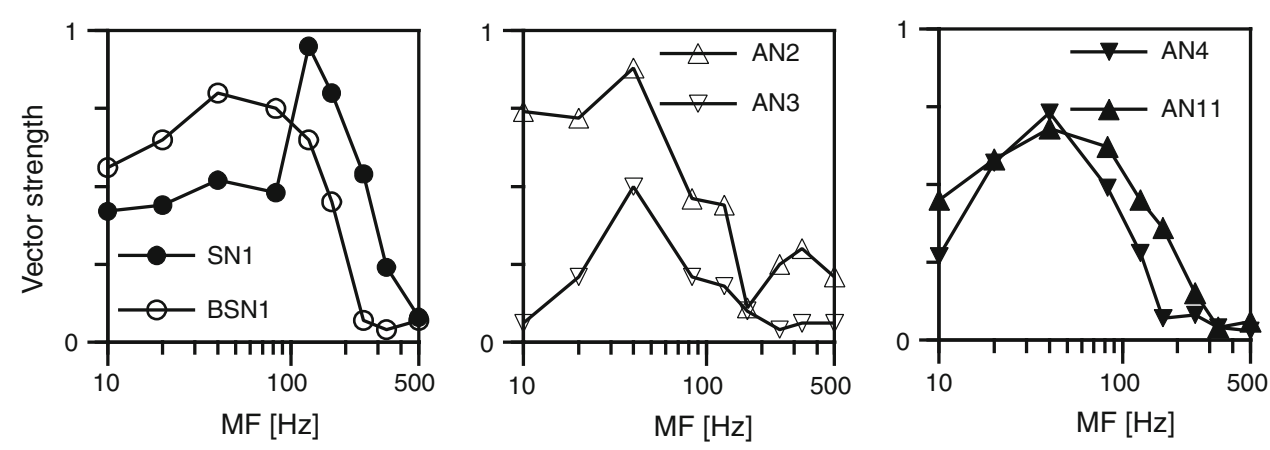

or bandpass behaviour (see Fig. 4; more examples are shown in supplementary Figs. 1, 2). Again, in order to summarize a large amount of data we extracted from the tMTF curves a few characteristic parameters: maximal vector strength, best modulation frequency (i.e. the frequency yielding the highest vector strength), and corner frequency (see inset in Fig. 5).

Most neurons $(86 \%)$ in our sample exhibited maximal vector strengths higher than 0.65 (Fig. 5a). A weakly significant decrease of max vector strength was observed between primary-like local and ascending neurons (ANOVA and Tukey-Kramer post hoc test: $P<0.05$; all other comparisons: n.s.). However, a pronounced difference between local and ascending neurons became visible in the plots of best modulation frequencies and corner frequencies, which were distinctly lower for ascending neurons than those of local and receptor neurons (Fig. 5b, c). The corner frequencies of ascending neurons were significantly lower than those of receptors and both classes of local neurons $(P<0.001, P<0.001, P<0.01)$, while all other combinations showed no significant differences (Kruskal-Wallis non parametric ANOVA and Dunn's post hoc test). The best modulation frequencies (Fig. 5b) of ascending neurons were significantly lower than those of receptors and primary-like LN (both $P<0.001$ ), but not significantly different from the class of 
Fig. 5 Summary diagram of tMTF data. a Maximal vector strengths; $R$ receptors, $L N$ local neurons, and $A N$ ascending neurons. b Best modulation frequencies, c corner frequencies (for definitions see inset)
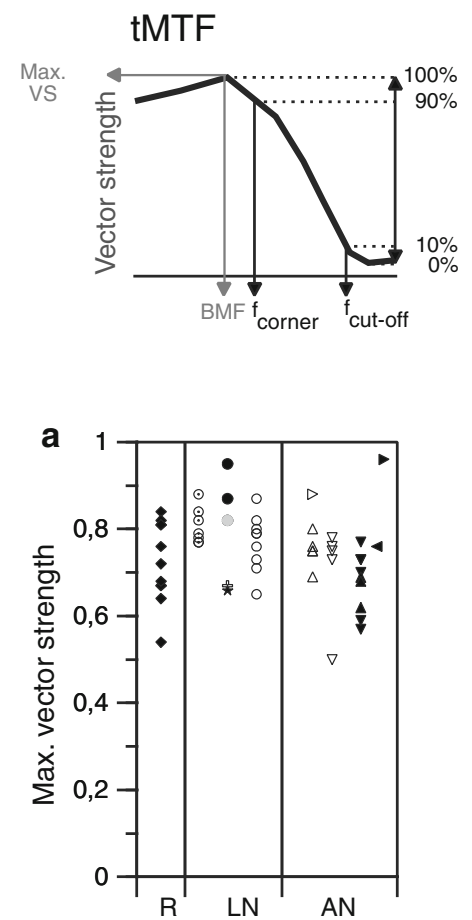

b

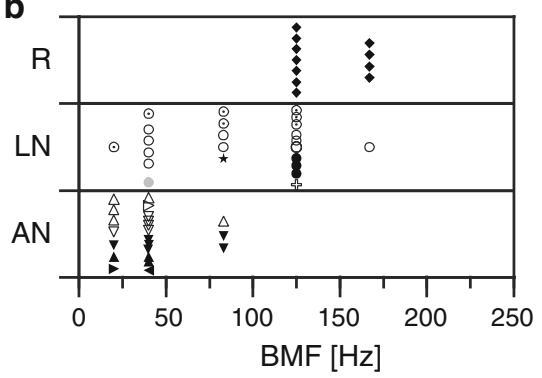

- Rec

$\odot$ TN1

- SN1

SN2

\& SN4

$\star$ SN8

○ BSN1

c

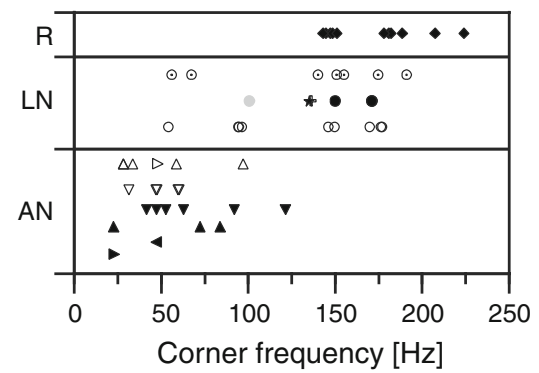

$\triangle \mathrm{AN1}$

$\triangleright$ AN2

$\nabla$ AN3

$\nabla$ AN4

A AN11

4 AN12

AN542 other local neurons (BSN1, SN2, and SN8). The latter differed from receptors $(P<0.05)$, while all other combinations exhibited no significant differences (KruskalWallis and Dunn's post hoc test). At the level of ascending neurons thus both the rMTF and the tMTF data indicate a restriction of processing power to the range of low modulation frequencies.

\section{Influence of modulation depth}

The data in Figs. 1, 2, 3, and 4 were obtained with $100 \%$ modulation depth. Figure 6a, left, shows that rMTF filter properties became less pronounced at reduced modulation depths. To classify deviations from an allpass rMTF, we used the criterion of a minimum spike rate difference of $40 \%$. With this criterion, 29 local and ascending neurons exhibited rate filter properties at $100 \%$ modulation depth. However, $48 \%$ of these lost their filter shape and changed to an allpass type when modulation depth was reduced to $50 \%$, and only $20 \%$ of the neurons preserved their filter characteristic at $25 \%$ modulation depth.

A similar effect was observed for the tMTFs; shallower modulations led to reduced vector strengths (Fig. 6a, right), and we found no indication for an enhanced modulation gain at the lowest modulation depth used. From the data like those shown in Fig. 6a, right, we calculated minimal modulation thresholds at the respective best modulation frequency. Minimal modulation thresholds of all primarylike local neurons exceeded $-25 \mathrm{~dB}$, i.e. these neurons produced a significant locking of spikes to the sound envelope with less than $6 \%$ modulation depth (see "Materials and methods" for definition). Modulation thresholds of the BSN1 specimens covered a broad range, between -15 and $-31 \mathrm{~dB}$ (corresponding to modulation depths between 18 and $2.8 \%$, respectively). Only 3 out of 9 BSN1 specimens and only 6 out of 20 ascending neurons exceeded a modulation threshold of $-25 \mathrm{~dB}$. Thus, the majority of BSN1 and most ascending neurons were insensitive to modulation depths smaller than $6 \%$.

\section{Influence of amplitude rise time}

We compared sinusoidal modulations (SAM) and modulations with rectangular rise times (RAM) in the same modulation frequency range (see "Materials and methods"). Some neurons that reacted differently to these two stimulation protocols were also tested with two intermediate rise times (see inset in Fig. 7). For most neurons, the rMTF filter shape changed only marginally when measured with RAM stimuli instead of SAM stimuli (see Fig. 7a, b): for all three receptors tested, five out of seven local neurons, and five out of eight ascending neurons, the maximal change in spike rate observed between SAM and RAM stimuli was less than $40 \%$. Two neurons whose responses changed drastically are shown in Fig. 7c, d. The responses of the local neuron SN8 (Fig. 7c) changed from a bandstop filter behaviour with SAM to a highpass filter with RAM stimuli. This was due to an inhibition induced by steep 
Fig. 6 a Influence of modulation depth on rMTF and tMTF of two representative neurons. b Modulation thresholds (in $\mathrm{dB}$ ) for various neurons. For the definition of modulation threshold see "Materials and methods". Note that a more negative $d B$ value indicates a better resolution of modulation depth. A nonparametric ANOVA revealed highly significant differences $(P=0.0094)$ between the neuron classes; however, Dunn's post hoc test indicates a significant difference only between ascending neurons and primary-like $\mathrm{LN}(P<0.01)$, all other combinations: n.s.
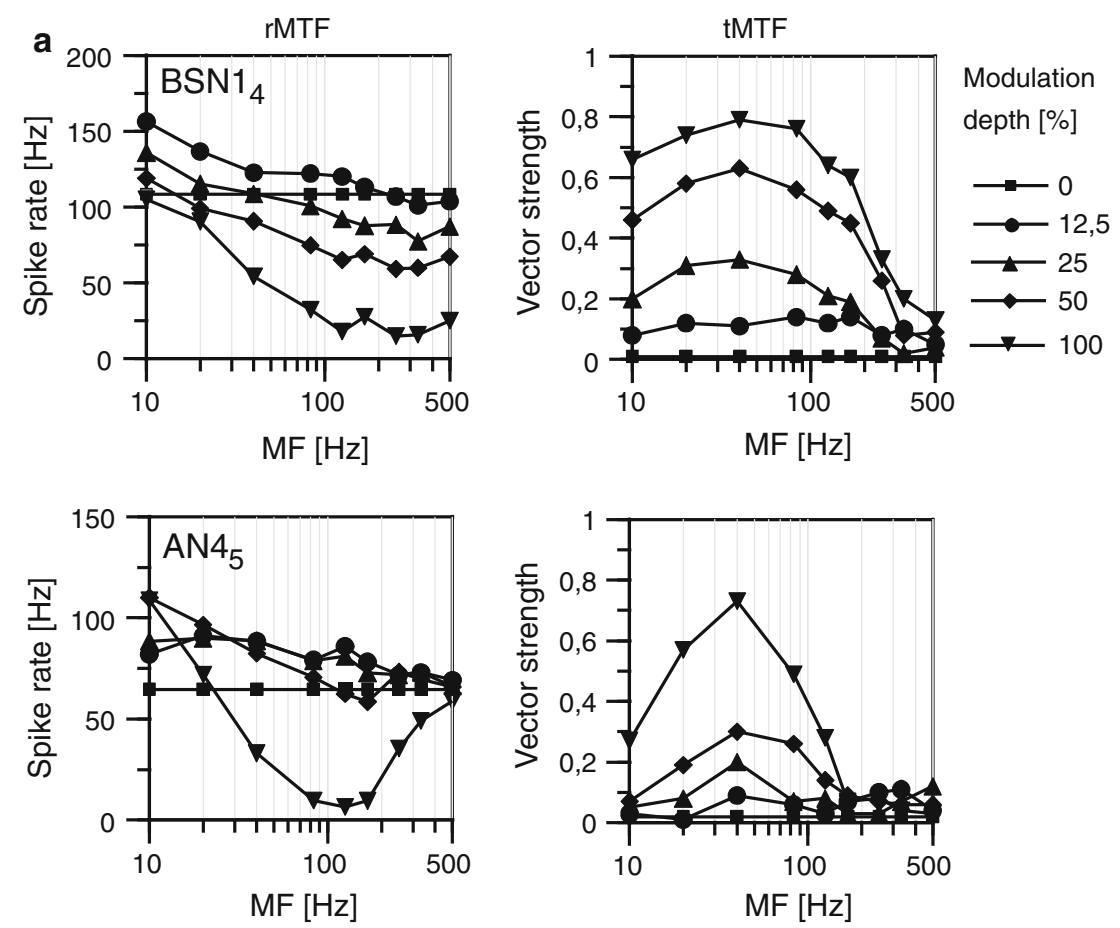

b

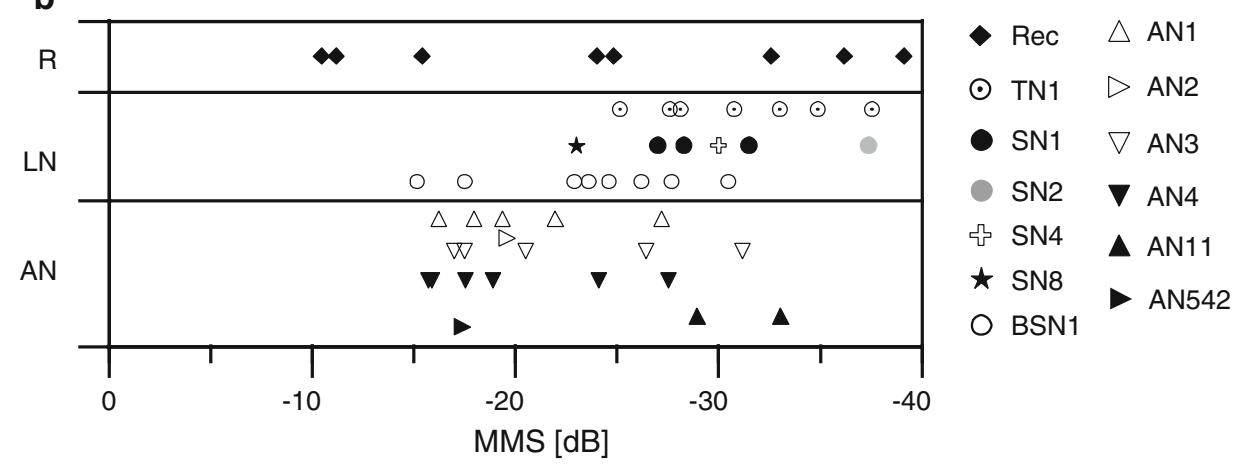

onsets at low modulation frequencies (Fig. 7e). A similarly prominent change was observed with one specimen of AN1 (Fig. 7d), while other AN1 specimens were less affected by steep stimulus onsets. Unexpectedly, the shape of the AN4 rMTF filter did not change between 20 and $500 \mathrm{~Hz}$ in the RAM and SAM conditions (max deviation 20\%; Fig. 7b).

The different onset slopes of the RAM and SAM stimuli had a modest influence on the shape of the tMTF curves of most neurons (Fig. 8a). With few exceptions (see Fig. 8b), best modulation frequencies and corner frequencies of neurons cluster around the diagonal in Fig. 8c, d. There was a significant trend towards a better resolution of AM depth in the RAM condition (minimal modulations thresholds: $-27.4 \pm 6.5$ compared to $-24.5 \pm 6.1, \quad P=0.0012$, mean $\pm \mathrm{SD}$; Wilcoxon paired test, two tailed), i.e. with a steeply rising stimulus onset already somewhat shallower modulations led to a significant locking of spikes to the stimulus envelope; the corresponding differences are, however, biologically probably not very relevant: minimal modulations depths of $4.3 \%$ (RAM) and $5.9 \%$ (SAM). The combined message of Figs. 7 and 8 therefore is that the tMTF filter functions of most neurons were only marginally affected by the onset steepness. The shapes of the rMTF functions of most auditory interneurons showed also no great differences between the SAM and RAM stimulation paradigms; there were, however, a few interesting exceptions (see Fig. 7c, d).

\section{Discussion}

In this study, we investigated the temporal processing capacities of auditory neurons of the locust at the first three stages of processing. The metathoracic ganglion houses an important stage of preprocessing, the output of which is transmitted to decision centres located in the 
Fig. 7 Influence of stimulus rise time on rMTF curves. a-d black symbols rectangular amplitude modulations (RAM); open symbols SAM stimuli. In c also two intermediate rise times were used (see inset and "Materials and methods"). e Morphology of the local neuron SN8 and sample recordings for SAM and RAM stimuli. Arrows indicate inhibitory events that lead to a suppression of spikes with low frequency RAM stimuli
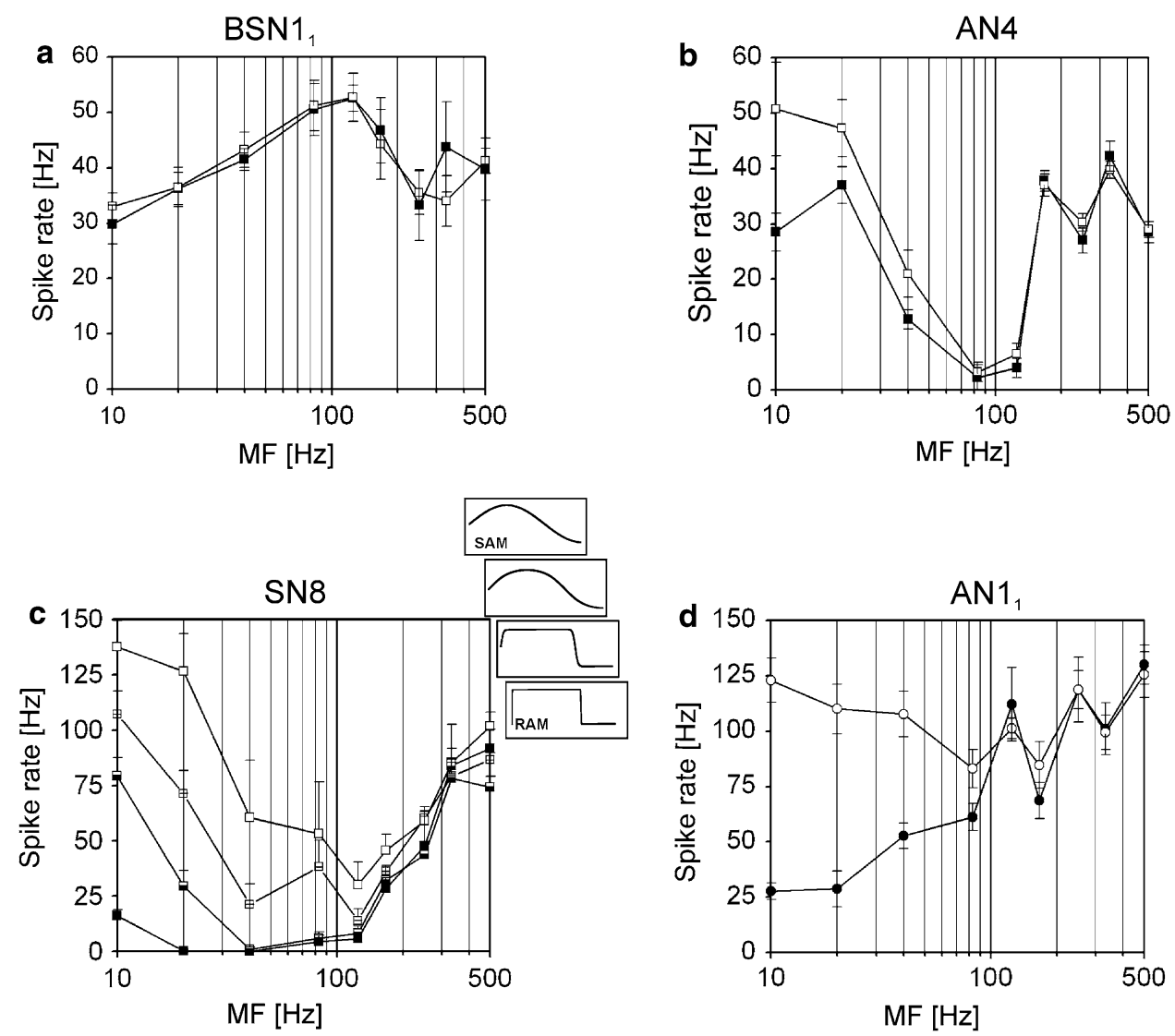

e

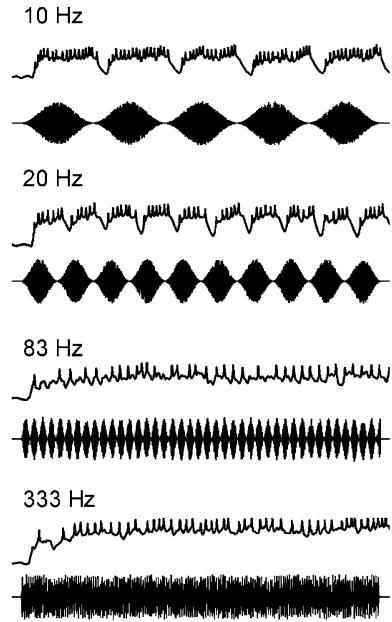

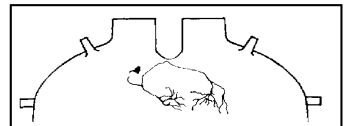
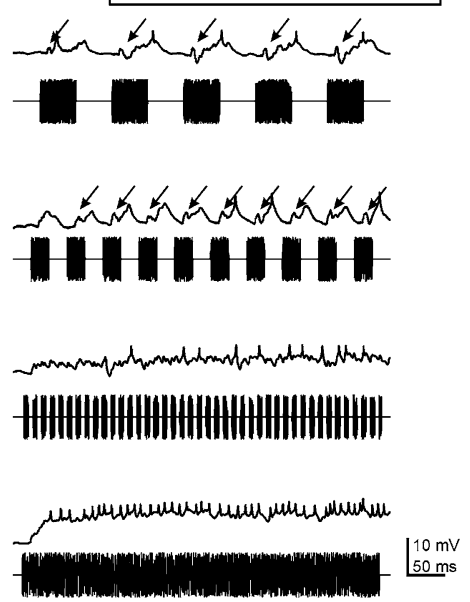

brain via ascending neurons (Ronacher et al. 1986; Bauer and von Helversen 1987). Due to size restrictions of insect nervous systems, we can expect that processing steps that are handled by large populations of neurons in the vertebrate brain may be concentrated onto a few or even single neurons in grasshoppers (see e.g. Ronacher and Stumpner 1988; Creutzig et al. 2009, 2010). Using a modulation transfer function paradigm we focused on three topics: (1) What are the limits of temporal resolution at different stages of processing, and how is information about different modulation frequencies transmitted to the brain? (2) What is the minimal modulation depth that can be resolved; how does it depend on the amplitude rise time? (3) To what degree does onset steepness affect the shape of modulation frequency filters? 
Fig. 8 Influence of stimulus rise time on tMTF curves. a, b Two sample neurons; c, $\mathbf{d}$ comparison of best modulation frequencies and corner frequencies for the SAM and RAM stimuli. For some neurons, data at two different intensities are included
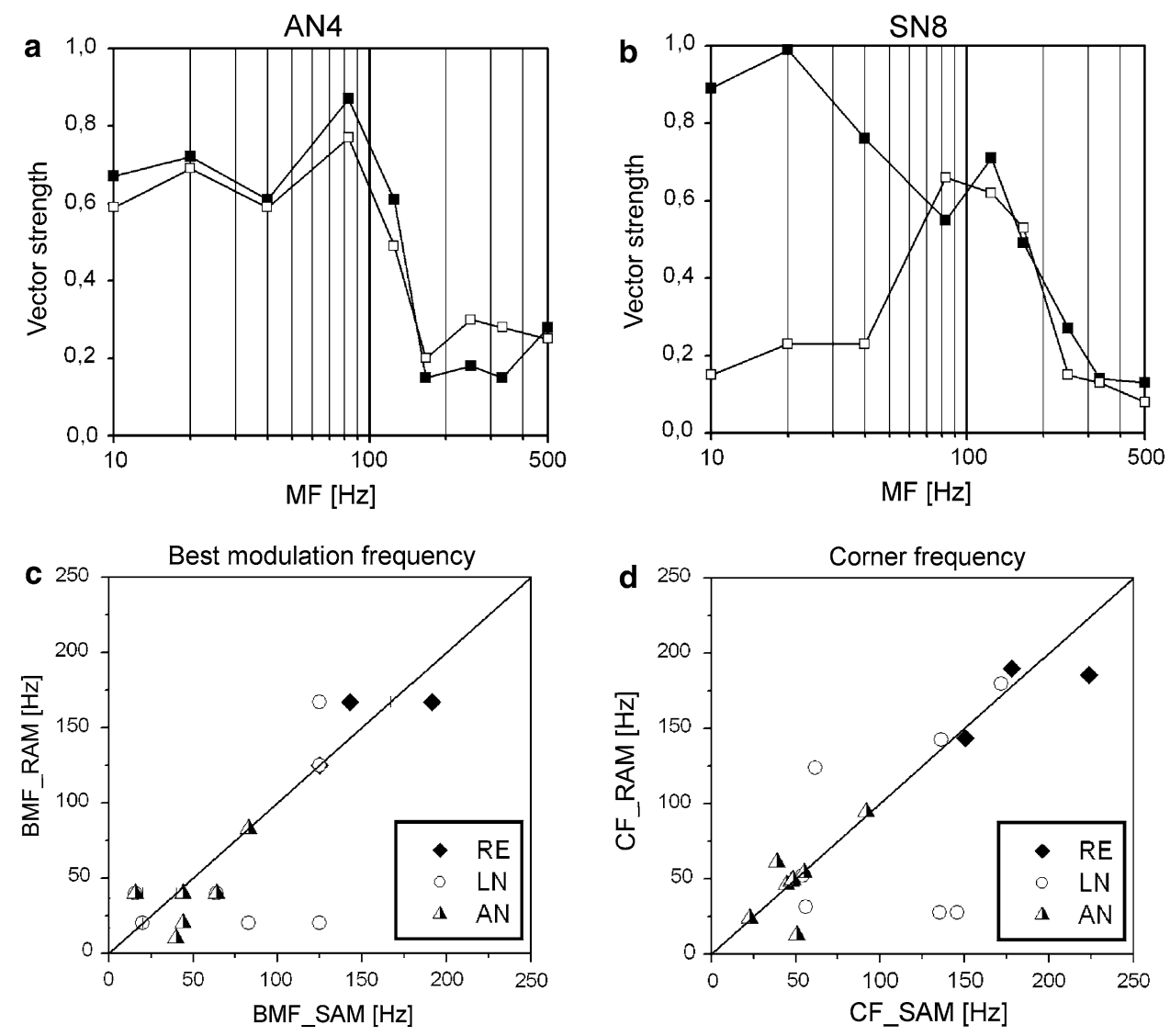

\section{Processing of modulation frequencies along the auditory pathway}

At the level of auditory receptors and primary-like neurons as TN1 and SN1, we found no spike rate changes by which specific AM frequency ranges could reliably be inferred. Such allpass behaviour appears to be a common feature of auditory receptor fibres also in vertebrates (see Rhode and Greenberg 1994; Joris et al. 2004). These neurons "reflect" the signal envelope in their spike pattern, and the timing of spikes becomes crucial for information transmission (see e.g. Rokem et al. 2006). In contrast to receptors and neurons with primary-like responses other local neurons, in particular BSN1, and most ascending neurons showed clear filter properties in their rMTF. The BSN1 neuron occurs as twin cells (Römer and Marquart 1984; Stumpner 1989) and is considered an important distributor of information within the auditory pathway (Boyan 1992). The rMTF of BSN1 specimens showed lowpass or bandpass properties while the rMTF of ascending neurons belonged mostly to the lowpass, bandpass or bandstop type (Fig. 2; supplementary Figs. 1, 2). The dynamic ranges of local neurons tended to be larger and to extend to higher modulation frequencies compared to that of the next processing stage, the ascending neurons (Fig. 3).
Due to their smaller dynamic range, a change of the spike rate of an ascending neuron could signal a more restricted AM-frequency segment for a read-out neuron than do local neurons-at least provided that some independent information, by other neurons, is available about the signal's intensity. However, the dynamic ranges of the rMTF of ascending neurons overlap to a high degree (Fig. 3b), making a range fractionation in the representation of modulation frequencies unlikely. Thus, we found no indication that a filter bank for modulation frequencies may exist in the locust as has been proposed for the auditory system of mammals (Langner and Schreiner 1988; Joris et al. 2004). Considering the strong conservation of the peripheral auditory pathway (Neuhofer et al. 2008), it seems not likely that the auditory system of $C$. biguttulus differs from locusts in that respect. The lack of a filter bank for AM frequencies is consistent with behavioural results in C. biguttulus that provide evidence against a Fourier analysis of sound envelopes, i.e. against a processing in the frequency domain (von Helversen and von Helversen 1998; Schmidt et al. 2008).

Spike timing of ascending neurons was found to be less precise compared to receptors or local neurons (Vogel et al. 2005; Wohlgemuth and Ronacher 2007). In view of these results, the high maximal vector strengths observed in the 
tMTFs of most ascending neurons were not expected (Fig. 5a). Such high values might result from the more phasic response properties of the ascending neurons (Stumpner and Ronacher 1991). However, with two other properties of the tMTF curves, a strong difference became evident between local and ascending neurons. A strong bias towards low modulation frequencies was observed in the tMTF data of ascending neurons, similar as for the rMTF data. Best modulation frequencies and corner frequencies were clearly shifted to low frequencies, compared to that of local neurons (Fig. 5b, c). There was a strong positive correlation between best modulation frequency and spike rate for receptors and local neurons $(r=0.84, P=0.0012$; $r=0.90, P<0.0001$, respectively), while for ascending neurons there was no correlation at all $(r=-0.02$, $P=0.93$; data not shown). This observation is in accord with the results of Weschke and Ronacher (2008).

Combining the results of the rMTF and tMTFdata evaluation, among most ascending neurons we observe a concentration of processing power to frequencies below $100 \mathrm{~Hz}$ and, compared to local neurons, a markedly reduced capacity to resolve high modulation frequencies. A similar restriction at higher processing stages to the range of low AM frequencies has also been reported for the auditory pathway of mammals (Malone et al. 2010). These features impose constraints upon the processing of temporal pattern information in grasshoppers since ascending neurons form a bottleneck for the information transmission to the brain.

In this context, it has to be emphasized that combinations of modulation frequencies between 10 and $50 \mathrm{~Hz}$ yield highly attractive song models for C. biguttulus females (von Helversen and von Helversen 1998; Schmidt et al. 2008), and that this frequency range plus the range between 60 and $120 \mathrm{~Hz}$ is also important for species discrimination (Clemens et al. 2010; Safi et al. 2006). However, further behavioural experiments on C. biguttulus females showed that modulation frequencies of 150-200 Hz may completely destroy the attractiveness of a song model (von Helversen and von Helversen 1998). The females detect tiny gaps, of only $2-3 \mathrm{~ms}$ width, in the signals of males that have lost one hindleg (von Helversen 1972; von Helversen and von Helversen 1997; see also Kriegbaum 1989). How can we reconcile these observations with the very low corner frequencies of ascending neurons, distinctly below $100 \mathrm{~Hz}$ (Fig. 5c), and their more restricted dynamic ranges (Fig. 3b)? Here, the bandstop filter properties come into play, which are exhibited by several types of ascending neurons (Figs. 2, 3b; supplementary Fig. 2). By a reduction of their spiking, such neurons could signal the presence of high modulation frequencies, up to $\sim 200 \mathrm{~Hz}$-again provided that additional information about the signal's SPL is available through other neurons. The AN4 neuron, for example, exhibits a strong bandstop property in its rMTF. Its spike response is almost completely inhibited at modulation frequencies between 80 and $200 \mathrm{~Hz}$ (Fig. 2; supplementary Fig. 2). This neuron is thought to mediate the remarkable gap detection response of $C$. biguttulus females (von Helversen 1972; Ronacher and Stumpner 1988; Stumpner et al. 1991; Franz and Ronacher 2002). Interestingly, for most of the bandstop neurons the range of spike suppression lies between 80 and $200 \mathrm{~Hz}$. This range coincides well with the behaviourally relevant range in C. biguttulus (von Helversen and von Helversen 1998) and the modulation frequencies relevant for the discrimination of songs of different gomphocerine grasshopper species (Safi et al. 2006; Clemens et al. 2010). Still higher modulation frequencies could not be resolved by the ascending neurons in our sample. The neurons with bandstop properties responded to SAM stimuli in the high frequency range in a similar way as to unmodulated noise.

Although some general trends appear if one compares local and ascending neurons, it is obvious that in particular the ascending neurons form a heterogeneous class with widely different response patterns (Römer and Marquart 1984; Stumpner and Ronacher 1991, 1994; Stumpner et al. 1991; Wohlgemuth and Ronacher 2007). In addition, a substantial variation of characteristic parameters exists even between the members of one neuron type (Figs. 3, 5; supplementary Figs. 1, 2). A similar amount of variation within one neuron type was found in C. biguttulus (Neuhofer et al. 2008, in preparation). This raises an interesting possibility: differences in the response of certain neuron types may lead to different response characteristics in behaving animals. Indeed, distinctly different response types were observed among C. biguttulus females (Balakrishnan et al. 2001; von Helversen et al. 2004). It would be worthwhile-but not easy-to correlate behavioural filter characteristics with neurophysiological recordings from the same individuals.

\section{Influence of modulation depth}

When discussing the rMTF filter properties of neurons at different stages of the auditory pathway, we cannot ignore the fact that the filter properties of most neurons become less pronounced for shallower modulation depths. Rate MTF were strongly affected by reduced modulation depths (Fig. 6). Half of the neurons' rMTF changed from a filter to allpass shape when the modulation depth was reduced to $50 \%$; at a modulation depth of $25 \%$ only $\sim 20 \%$ of the neurons retained their filter shape. Maximal vector strengths were also reduced at lower modulation depths (Fig. 6). During communication episodes under field 
conditions, the modulation depths of grasshopper songs will be reduced due to ambient noise and interfering sound sources like other singing grasshoppers (Römer 1998, 2001; Lang 2000; Gilbert and Elsner 2000). Thus, the neuronal filtering of modulation frequencies will probably be less efficient under natural conditions. This effect may contribute to the rather small actual communication distances observed in the field (Lang 2000).

\section{Influence of amplitude rise time}

Rise time of sound pulses is a cue for discrimination between male and female songs in C. biguttulus (von Helversen 1993; von Helversen and von Helversen 1997), and slowly rising ramps may support sound localization (Krahe and Ronacher 1993; Ronacher and Krahe 2000). Earlier evidence indicated that steep amplitude modulations enable more precise spiking (Krahe and Ronacher 1993; Machens et al. 2001; Rokem et al. 2006). Hence, we had expected that in the RAM condition the best modulation and corner frequencies of the tMTF curves would shift to higher frequencies. However, in most neurons we did not observe a corresponding change (Fig. 8c, d). Indeed, more neurons had higher best modulation and corner frequencies in the SAM condition. Moreover, only a minority of neurons (28\%) exhibited a marked change in their rMTF filter properties between SAM and RAM stimuli (the two most prominent changes are shown in Fig. 7c, d). In these neurons, the strongest differences between the RAM and SAM conditions occurred at low modulations frequencies $(10-40 \mathrm{~Hz})$, i.e. at frequencies with the largest differences in rise times between RAM and SAM stimuli.

In conclusion, the processing of stimulus envelope patterns occurring between receptor cells to ascending interneurons restricts the available information to the range of low modulation frequencies and clearly defined modulation depths. Since ascending neurons are the only source of information for decision centres located in the brain, we must ask how the animals are able to recognize conspecific song signals in spite of these limitations of their auditory pathway. However, at the level of ascending neurons, additional features may become more important than the pure filtering of AM frequencies: the spike rate filter properties for distinct features of the song, like presence of gaps (AN4 neuron; Ronacher and Stumpner 1988; Stumpner et al. 1991), or presence of pauses of a certain duration (AN12 neuron; Creutzig et al. 2009; 2010), features that proved to be relevant for signal discrimination in grasshoppers (e.g. von Helversen and von Helversen 1994, 1997). Remarkably, the song envelopes of many grasshopper species differ most in the range of low modulation frequencies (below $110 \mathrm{~Hz}$; Clemens et al. 2010; see also
Safi et al. 2006), and low AM frequencies were found to be most crucial for species recognition in behavioural tests (von Helversen and von Helversen 1998; Schmidt et al. 2008). The concentration of song features to the range of low modulation frequencies may have evolved primarily as a response to the conserved specific response properties of auditory neurons in grasshoppers (Neuhofer et al. 2008; Clemens et al. 2010). This feature of the auditory pathway of these insects in turn might reflect conditions of sound transmission in natural habitats. High modulation frequencies-as present, e.g. in a combination of short sound pulses and gaps, in the ms range-tend to be distorted during sound transmission due to scattering and reverberations (e.g. Fig. 4 in Michelsen and Larsen 1983; see also Michelsen et al. 1985; Michelsen and Rohrseitz 1997). This leaves low modulation frequencies as the more salient cues (see also Richards and Wiley 1980; Bradbury and Vehrencamp 1998).

Acknowledgments We thank two anonymous reviewers for their helpful comments. The study was supported by grants from the DFG (Sonderforschungsbereich 618) to B.R. The experiments comply with the current laws on "Principles of animal care" in Germany.

Open Access This article is distributed under the terms of the Creative Commons Attribution Noncommercial License which permits any noncommercial use, distribution, and reproduction in any medium, provided the original author(s) and source are credited.

\section{References}

Balakrishnan R, von Helversen D, von Helversen O (2001) Song pattern recognition in the grasshopper Chorthippus biguttulus: the mechanism of syllable onset and offset detection. J Comp Physiol A 187:255-264

Bauer M, von Helversen O (1987) Separate localisation of sound recognizing and sound producing neural mechanisms in a grasshopper. J Comp Physiol A 165:687-695

Boyan GS (1992) Common synaptic drive to segmentally homologous interneurons in the locust. J Comp Neurol 321:544-554

Bradbury JW, Vehrencamp S (1998) Principles of animal communication. Sinauer Associates Inc., Massachusetts

Clemens J, Weschke G, Vogel A, Ronacher B (2010) Intensity invariance properties of auditory neurons compared to the statistics of relevant natural signals in grasshoppers. J Comp Physiol A 196:285-297

Creutzig F, Wohlgemuth S, Stumpner A, Benda J, Ronacher B, Herz AVM (2009) Time-scale invariant representation of acoustic communication signals by a bursting neuron. J Neurosci 29:2575-2580

Creutzig F, Benda J, Wohlgemuth S, Stumpner A, Ronacher B, Herz AVM (2010) Timescale-invariant pattern recognition by feedforward inhibition and parallel signal processing. Neural Comput 22:1493-1510

Elsner N (1974) Neuroethology of sound production in Gomphocerine grasshoppers (Orthoptera: Acrididae) 1 Song patterns and stridulatory movements. J Comp Physiol 88:67-102 
Franz A (2004) Neuronale Variabilität und Korrelationen als begrenzende Faktoren für die Verarbeitung und Kodierung zeitlich strukturierter akustischer Signale. PhD thesis, Humboldt-Universität zu Berlin

Franz A, Ronacher B (2002) Temperature dependence of time resolution in an insect nervous system. J Comp Physiol A 188:261-271

Gerhardt HC, Huber F (2002) Acoustic communication in insects and anurans. The University of Chicago Press, Chicago

Gilbert F, Elsner N (2000) Directional hearing of a grasshopper in the field. J Exp Biol 203:983-993

Gleich O, Klump GM (1995) Temporal modulation transfer functions in the European starling (Sturnus vulgaris): 2. Responses of auditory nerve fibers. Hear Res 82:81-92

Greenfield MD (2002) Signalers and receivers: mechanisms and evolution of arthropod communication. Oxford University Press, Oxford

Hall JC, Feng AS (1991) Temporal processing in the dorsal medullary nucleus of the northern leopard frog (Rana pipiens pipiens). J Neurophysiol 66:955-973

Heil P, Neubauer H (2001) Temporal integration of sound pressure determines thresholds of auditory-nerve fibers. J Neurosci 21:7404-7415

Heller K-G (1988) Bioakustik der europäischen Laubheuschrecken. Josef Margraf Verlag, Weikersheim, ISBN 3-8236-1165-6

Hennig RM, Franz A, Stumpner A (2004) Processing of auditory information in insects. Microsc Res Tech 63:351-374

Joris PX, Schreiner CE, Rees A (2004) Neural processing of amplitude-modulated sounds. Physiol Rev 84:541-577

Krahe R, Ronacher B (1993) Long rise times of sound pulses in grasshopper songs improve the directionality cues received by the CNS from the auditory receptors. J Comp Physiol A 173:425-434

Kriegbaum H (1989) Female choice in the grasshopper Chorthippus biguttulus. Naturwissenschaften 76:81-82

Krishna BS, Semple MN (2000) Auditory temporal processing: responses to sinusoidally amplitude-modulated tones in the inferior colliculus. J Neurophysiol 84:255-273

Kroodsma DE, Miller EH (1996) Ecology and evolution of acoustic communication in birds. Cornell University Press, Ithaca

Lang F (2000) Acoustic communication distances of a gomphocerine grasshopper. Bioacoustics 10:233-258

Langner G, Schreiner CE (1988) Periodicity coding in the inferior colliculus of the cat I. Neuronal mechanisms. J Neurophysiol 60:1799-1821

Machens CK, Stemmler MB, Prinz P, Krahe R, Ronacher B, Herz AVM (2001) Representation of acoustic communication signals by insect auditory receptor neurons. J Neurosci 21:32153227

Malone BJ, Scott BH, Semple MN (2010) Temporal codes for amplitude contrast in auditory cortex. J Neurosci 30:767-784

Meyer J, Elsner N (1996) How well are frequency sensitivities of grasshopper ears tuned to species-specific song spectra? J Exp Biol 199:1631-1642

Michelsen A, Larsen ON (1983) Strategies for acoustic communication in complex environments. In: Huber F, Markl H (eds) Neuroethology and behavioural physiology. Springer, Berlin, pp 321-331

Michelsen A, Rohrseitz K (1997) Sound localisation in a habitat: an analytical approach to quantifying the degradation of directional cues. Bioacoustics 7:291-313

Michelsen A, Larsen ON, Surlykke A (1985) Auditory processing of temporal cues in insect songs: frequency domain or time domain. In: Michelsen A (ed) Time resolution in auditory systems. Springer, New York, pp 3-27
Neuhofer D, Wohlgemuth S, Stumpner A, Ronacher B (2008) Evolutionarily conserved coding properties of auditory neurons across grasshopper species. Proc R Soc Lond B 208:1965-1974

Prinz P, Ronacher B (2002) Temporal modulation transfer functions in auditory receptor fibres of the locust (Locusta migratoria). J Comp Physiol A 188:577-587

Rhode WS, Greenberg S (1994) Encoding of amplitude modulation in the cochlear nucleus of the cat. J Neurophysiol 71:17971825

Richards DG, Wiley RH (1980) Reverberations and amplitude fluctuations in the propagation of sound in a forest: implications for animal communication. Am Nat 115:381-399

Rieke F, Warland D, van Steveninck R, Bialek W (1996) Spikesexploring the neural code. MIT Press, Cambridge

Rokem A, Watzl S, Gollisch T, Stemmler MB, Herz AVM, Samengo I (2006) Spike-timing precision underlies the coding efficiency of auditory receptor neurons. J Neurophysiol 95:2541-2552

Römer H (1998) The sensory ecology of acoustic communication in insects. In: Hoy RR, Popper AN, Fay RR (eds) Comparative hearing: insects. Springer, New York, pp 63-96

Römer H (2001) Ecological constraints for sound communication: from grasshoppers to elephants. In: Barth FG, Schmid A (eds) Ecology of sensing. Springer, Berlin, pp 59-77

Römer H, Marquart V (1984) Morphology and physiology of auditory interneurons in the metathoracic ganglion of the locust. J Comp Physiol A 155:249-262

Ronacher B, Krahe R (2000) Temporal integration vs. parallel processing: coping with the variability of neuronal messages in directional hearing of insects. Eur J Neurosci 12:2147-2156

Ronacher B, Stumpner A (1988) Filtering of behavioural relevant parameters of a grasshopper's song by an auditory interneuron. J Comp Physiol A 163:517-523

Ronacher B, von Helversen D, von Helversen O (1986) Routes and stations in the processing of auditory directional information in the CNS of a grasshopper, as revealed by surgical experiments. J Comp Physiol A 158:363-374

Safi K, Heinzle J, Reinhold K (2006) Species recognition influences female mate preferences in the common European grasshopper (Chorthippus biguttulus L.). Ethology 112:1225-1230

Schmidt A, Ronacher B, Hennig RM (2008) The role of frequency, phase and time for processing amplitude modulated signals by grasshoppers. J Comp Physiol A 194:221-233

Sokoliuk T, Stumpner A, Ronacher B (1989) GABA-like immunoreactivity suggests an inhibitory function of the thoracic-lowfrequency-neuron (TN1) in grasshoppers. Naturwissenschaften $76: 223-225$

Stumpner A (1989) Physiological variability of auditory neurons in a grasshopper. Naturwissenschaften 76:427-429

Stumpner A, Ronacher B (1991) Auditory interneurons in the metathoracic ganglion of the grasshopper Chorthippus biguttulus. 1. Morphological and physiological characterization. J Exp Biol 158:391-410

Stumpner A, Ronacher B (1994) Neurophysiological aspects of song pattern recognition and sound localization in grasshoppers. Am Zool 34:696-705

Stumpner A, von Helversen O (1994) Song production and song recognition in a group of sibling grasshopper species (Chorthippus dorsatus, C. dichrous, and C. loratus: Orthoptera, Acrididae). Bioacoustics 6:1-23

Stumpner A, von Helversen D (2001) Evolution and function of auditory systems in insects. Naturwissenschaften 88:159-170

Stumpner A, Ronacher B, von Helversen O (1991) Auditory interneurons in the metathoracic ganglion of the grasshopper Chorthippus biguttulus. 2. Processing of temporal patterns of the song of the male. J Exp Biol 158:411-430 
Vogel A, Ronacher B (2007) Neural correlations increase between consecutive processing levels in the auditory system of locusts. J Neurophysiol 97:3376-3385

Vogel A, Hennig RM, Ronacher B (2005) Increase of neuronal response variability at higher processing levels as revealed by simultaneous recordings. J Neurophysiol 93:3548-3559

von Helversen D (1972) Gesang des Männchens und Lautschema des Weibchens bei der Feldheuschrecke Chorthippus biguttulus (Orthoptera, Acrididae). J Comp Physiol A 81:381-422

von Helversen D (1993) 'Absolute steepness' of ramps as an essential cue for auditory pattern recognition by a grasshopper (Orthoptera, Acrididae Chorthippus biguttulus L.). J Comp Physiol A 172:633-639

von Helversen D, von Helversen O (1997) Recognition of sex in the acoustic communication of the grasshopper Chorthippus biguttulus (Orthoptera, Acrididae). J Comp Physiol A 180:373-386 von Helversen D, von Helversen O (1998) Acoustic pattern recognition in a grasshopper: processing in the frequency or time domain? Biol Cybern 79:467-476

von Helversen D, Balakrishnan R, von Helversen O (2004) Acoustic communication in a duetting grasshopper: receiver response variability, male strategies and signal design. Anim Behav 68:131-144

von Helversen O, von Helversen D (1994) Forces driving coevolution of song and song recognition in grasshoppers. In: Schildberger $\mathrm{K}$, Elsner $\mathrm{N}$ (eds) Neural basis of behavioural adaptations. G. Fischer Verlag, Stuttgart, pp 253-284

Weschke G, Ronacher B (2008) Influence of sound pressure level on the processing of amplitude modulations by auditory neurons of the locust. J Comp Physiol A 194:255-265

Wohlgemuth S, Ronacher B (2007) Auditory discrimination of amplitude modulations based on metric distances of spike trains. J Neurophysiol 97:3082-3092 